Gudrun Szewieczek (1)

\title{
A duality for Guichard nets
}

Received: 25 April 2019 / Accepted: 10 February 2020 /

Published online: 26 February 2020

\begin{abstract}
In this paper we study G-surfaces, a rather unknown surface class originally defined by Calapso, and show that the coordinate surfaces of a Guichard net are G-surfaces. Based on this observation, we present distinguished Combescure transformations that provide a duality for Guichard nets. Another class of special Combescure transformations is then used to construct a Bäcklund-type transformation for Guichard nets. In this realm a permutability theorem for the dual systems is proven.
\end{abstract}

\section{Introduction}

Various integrable surface classes were classically characterized by the existence of particular Combescure transformed dual surfaces: for example, isothermic surfaces via the Christoffel transforms and Guichard surfaces by the Guichard duality ([5,8, $15,18]$ ). Based on this principle, in [27] the theory of O-surfaces was established, which provides a unified way to describe many classically known integrable surface classes.

Recently [25], a generalization of Demoulin's duality for $\Omega$-surfaces [12] has been found, revealing that the Lie applicable class of $\Omega$-surfaces also appears as Osurfaces. Moreover, this concept also applies to higher dimensional hypersurfaces as the example of 3-dimensional conformally flat hypersurfaces shows (cf. [23,29]). Although triply orthogonal systems possess a rich transformation theory ([2,3, $11,17])$, the concept of characterizing Combescure transformations to distinguish special subclasses of systems has not been exploited yet. In this paper we adopt this approach and present a duality for the subclass of Guichard nets that allows, together with particular associated systems, a characterization of Guichard nets in terms of their Combescure transforms.

Guichard nets, that is, triply orthogonal systems with an induced metric fulfilling a special trace-zero-condition, were commonly studied by classical geometers around $1900([11,19,26])$. Renewed interest arises from the observation that Guichard nets provide characterizing curvature line coordinates for 3-dimensional conformally flat hypersurfaces [20]. This has created recent research interest at the junction of these triply orthogonal systems and hypersurfaces $([4,7,13,14,30])$. In particular,

G. Szewieczek ( $\varangle)$ : TU Wien, Wiedner Hauptstr. 8-10/104, 1040 Vienna, Austria. e-mail: gudrun@geometrie.tuwien.ac.at

Mathematics Subject Classification: 53A05 · 53A30 - 37K25 · 37K35

https://doi.org/10.1007/s00229-020-01181-7 
special subclasses such as cyclic Guichard nets [21,24], Bianchi-type Guichard nets [22] and isothermic Guichard nets [28,29] have been investigated.

The duality for Guichard nets presented in this paper relies on the crucial fact that the coordinate surfaces of a Guichard net are G-surfaces. This surface class was introduced by Calapso in [6] and then apparently fell into oblivion: a surface $f$ is a G-surface if there exists a Combescure related associated surface $\hat{f}$ such that the following relation

$$
c H_{1}^{2} H_{2}^{2}\left(\frac{\kappa_{1}}{\hat{\kappa}_{1}}-\frac{\kappa_{2}}{\hat{\kappa}_{2}}\right)^{2}=H_{2}^{2}+\varepsilon H_{1}^{2} \text { for some } \varepsilon \in\{0, \pm 1\} \text { and } c \in \mathbb{R} \backslash\{0\}
$$

between the principal curvatures and the coefficients $H_{1}^{2}$ and $H_{2}^{2}$ of the induced metric of $f$ holds. Note, however, that this relation is not symmetric in $f$ and $\hat{f}$, hence the Combescure transform $\hat{f}$ is in general not a G-surface with associated surface $f$.

In Sect. 3 we demonstrate that for a G-surface there additionally exist Combescure transforms that are again G-surfaces and allow for a dual construction. This characterization immediately reveals that G-surfaces can be interpreted in the framework of O-surfaces and contain isothermic, as well as Guichard surfaces as subclasses.

These observations will be used in Sect. 4 to prove how G-surfaces arise in the context of triply orthogonal systems. In particular, we show that the coordinate surfaces of a Guichard net are G-surfaces. As a consequence, there exist associated and dual surfaces for the coordinate surfaces of a Guichard net that, suitably chosen, give rise to new triply orthogonal systems. It is proven that the triply orthogonal systems formed by the dual surfaces are again Guichard nets, hence this dual construction provides a method to construct new Guichard nets from a given one.

Another way of obtaining new Guichard nets was given in [2,3]: it has been shown that for any Guichard net there exist Ribaucour transformations that preserve the Guichard condition. We reconsider this Bäcklund-type transformation in Sect. 5 by using the fact that any Ribaucour transformation can be decomposed into two Combescure transformations and an inversion in the unit sphere. In this way, the Ribaucour transformation between two Guichard nets is traced back to the determination of particular Combescure transforms of a Guichard net. Moreover, we prove permutability between this Ribaucour transformation and the duality for Guichard nets.

\section{Preliminaries}

In this work we only deal with surfaces $f=\left(f_{1}, f_{2}, f_{3}\right): M^{2} \rightarrow \mathbb{R}^{3}$ parametrized by curvature line coordinates, that is, the coordinates are orthogonal and conjugate. Equivalently, the coordinate functions $f_{1}, f_{2}$ and $f_{3}$, as well as the function $|f|^{2}$, are solutions of the point equation of the surface [15, Chap. I.2, IV.61]

$$
\partial_{x y} \theta=\partial_{y} \log H_{1} \partial_{x} \theta+\partial_{x} \log H_{2} \partial_{y} \theta,
$$

where the induced metric of the surface is denoted by $I=H_{1}^{2} d x^{2}+H_{2}^{2} d y^{2}$. 


\subsection{Combescure transformation of a surface}

Classically $[9,15]$, two surfaces $f$ and $\hat{f}$ are related by a Combescure transformation if they have parallel tangent planes along corresponding conjugate coordinate lines. However, note that a Combescure transformation generically preserves curvature line coordinates. Hence, in this paper we consider without loss of generality parallel tangent directions along curvature line coordinates.

Analytically, any Combescure transform $\hat{f}$ of $f: M^{2} \rightarrow \mathbb{R}^{3}$ is (up to translation) uniquely determined by

$$
d \hat{f}=h \partial_{x} f d x+l \partial_{y} f d y
$$

where $h, l: M^{2} \rightarrow \mathbb{R}$ are two functions fulfilling the compatibility conditions

$$
\begin{aligned}
\partial_{y} h & =(l-h) \partial_{y} \ln H_{1}, \\
\partial_{x} l & =(h-l) \partial_{x} \ln H_{2} .
\end{aligned}
$$

Equivalently [15, Chap. I.4], any function $\varphi: M^{2} \rightarrow \mathbb{R}$ fulfilling

$$
\partial_{x y} \varphi+\partial_{y} \ln H_{1} \partial_{x} \varphi+\partial_{x} \ln H_{2} \partial_{y} \varphi+\varphi \partial_{x y} \ln \left(H_{1} H_{2}\right)=0
$$

gives rise to a pair of functions $h$ and $l$ described by

$$
\begin{aligned}
\partial_{x} h & =\varphi \partial_{x} \ln \left(H_{2} \varphi\right), \\
\partial_{y} h & =-\varphi \partial_{y} \ln H_{1}, \\
l & =h-\varphi,
\end{aligned}
$$

that fulfills equations (3) and therefore defines by (2) a Combescure transformation of $f$.

Since the functions $h$ and $l$ are uniquely determined up to the same additive constant, a function $\varphi$ satisfying condition (4) induces by (5) a 1-parameter family of Combescure transforms of $f$.

Note that, in particular, the normals of $f$ and a Combescure transform $\hat{f}$ coincide up to orientation, that is, $n=\delta \hat{n}$, where $\delta \in\{ \pm 1\}$ depending on whether the product $h l$ is positive or negative. Hence, denoting the principal curvatures of $f$ by $\kappa_{1}$ and $\kappa_{2}$, the principal curvatures $\hat{\kappa}_{1}$ and $\hat{\kappa}_{2}$ of the Combescure transform $\hat{f}$ are given by

$$
\hat{\kappa}_{1}=\delta \frac{\kappa_{1}}{h} \text { and } \hat{\kappa}_{2}=\delta \frac{\kappa_{2}}{l}
$$

We also allow the functions $h$ or $l$ to vanish identically, such that $\hat{f}$ degenerates to a curve or a point. In these cases we consider the corresponding radii of principal curvature of $\hat{f}$ to be zero. 


\subsection{Triply orthogonal systems and their combescure transforms}

In what follows, we give a brief introduction to triply orthogonal systems and summarize some facts that will become important later. We then focus on the Combescure transformation for triply orthogonal systems and prove two results that show how these transforms can be constructed.

For more details and a wide range of beautiful results in this area, we refer to the rich literature on triply orthogonal systems, for example [1,11,24,26,31].

Agreement. Throughout the paper we use the following convention for the indices in the realm of triply orthogonal systems: if only one index $i$ or two indices $i$ and $j$ appear in an equation, then $i \neq j \in\{1,2,3\}$. If there are three indices $(i, j, k)$ involved, then they take the values $(1,2,3)$ or a cyclic permutation of them.

To avoid useless indices, we sometimes change the notations of variables: $x=$ $x_{1}, x_{2}=y$ and $x_{3}=z$. For the partial derivatives we use the abbreviations $\partial_{i}=\partial_{x_{i}}$ and $\partial_{i j}=\partial_{x_{i}} \partial_{x_{j}}$.

Let $U$ be an open and connected subset of $\mathbb{R}^{3}$, then $f: U \rightarrow \mathbb{R}^{3}$ is a triply orthogonal system if $\operatorname{det}\left(\partial_{x} f, \partial_{y} f, \partial_{z} f\right) \neq 0$ and

$$
\left(\partial_{i} f, \partial_{j} f\right)=0
$$

where $(.,$.$) denotes the usual inner product on \mathbb{R}^{3}$. The unit normals $N_{i}$ of the coordinate surfaces $x_{i}=$ const are given by

$$
N_{i}:=\frac{\partial_{j} f \times \partial_{k} f}{\left|\partial_{j} f \times \partial_{k} f\right|}
$$

and the functions $H_{1}, H_{2}, H_{3}: U \rightarrow \mathbb{R}$ defined by

$$
\partial_{i} f=H_{i} N_{i}
$$

are called the Lamé coefficients of $f$. Since

$$
\operatorname{det}\left(\partial_{x} f, \partial_{y} f, \partial_{z} f\right)=H_{1} H_{2} H_{3},
$$

the Lamé coefficients never vanish on $U$. The induced metric of a triply orthogonal system is then given by

$$
I=(d f, d f)=H_{1}^{2} d x^{2}+H_{2}^{2} d y^{2}+H_{3}^{2} d z^{2} .
$$

If $f$ is a triply orthogonal system, then the functions $\left(f_{1}, f_{2}, f_{3}\right)=f$ are solutions of the point equations of any surface family $x_{k}=$ const,

$$
\partial_{i j} f=\partial_{j} \ln H_{i} \partial_{i} f+\partial_{i} \ln H_{j} \partial_{j} f
$$

and fulfill the Gauss equation

$$
\partial_{i}^{2} f=\partial_{i} \ln H_{i} \partial_{i} f-\frac{H_{i}}{H_{j}^{2}} \partial_{j} H_{i} \partial_{j} f-\frac{H_{i}}{H_{k}^{2}} \partial_{k} H_{i} \partial_{k} f .
$$


Moreover, the Lamé coefficients $H_{1}, H_{2}$ and $H_{3}$ satisfy the first and second system of Lamé's equations

$$
\begin{aligned}
\partial_{i j} H_{k} & =\partial_{j} \ln H_{i} \partial_{i} H_{k}+\partial_{i} \ln H_{j} \partial_{j} H_{k}, \\
0 & =\frac{1}{H_{k}^{2}} \partial_{k} H_{i} \partial_{k} H_{j}+\partial_{j}\left\{\frac{1}{H_{j}} \partial_{j} H_{i}\right\}+\partial_{i}\left\{\frac{1}{H_{i}} \partial_{i} H_{j}\right\} .
\end{aligned}
$$

If we introduce Darboux's rotational coefficients

$$
\beta_{i j}:=\frac{1}{H_{i}} \partial_{i} H_{j},
$$

then Lamé's systems (8) and (9) reduce to first order equations

$$
\begin{aligned}
& 0=\partial_{k} \beta_{i j}-\beta_{i k} \beta_{k j}, \\
& 0=\partial_{i} \beta_{i j}+\partial_{j} \beta_{j i}+\beta_{k i} \beta_{k j}
\end{aligned}
$$

and the unit normals $N_{1}, N_{2}$ and $N_{3}$ satisfy the system

$$
\begin{aligned}
\partial_{j} N_{i} & =\beta_{i j} N_{j}, \\
\partial_{i} N_{i} & =-\beta_{j i} N_{j}-\beta_{k i} N_{k}, \\
\left(N_{i}, N_{j}\right) & =\delta_{i j} .
\end{aligned}
$$

Conversely, an orthogonal metric of the form (6) that satisfies Lamé's equations gives rise to a triply orthogonal system, which is uniquely determined up to Euclidean motions: to recover a parametrization $f: U \rightarrow \mathbb{R}^{3}$ from a prescribed orthogonal metric $I$, we first compute the rotational coefficients $\beta_{i j}$ and construct a set of suitable normals $N_{1}, N_{2}$ and $N_{3}$ from system (10). Then a parametrization $f: U \rightarrow \mathbb{R}^{3}$ of the system with induced metric $I$ is obtained by integrating

$$
d f=H_{1} N_{1} d x+H_{2} N_{2} d y+H_{3} N_{3} d z .
$$

Note that the integrability of the system (10) and Eq. (11) is guaranteed by Lamé's equations and the construction is unique up to Euclidean motions.

Thus, if the ambiguity of Euclidean motions does not effect the geometry we are interested in, we talk about the induced metric instead of the explicit parametrization.

Recall that, by Dupin's Theorem, any two coordinate surfaces of two different families intersect along a curve which is a curvature line for both surfaces. This immediately reveals information about the geometry of the three families of coordinate surfaces: the induced metrics $I_{i}$ for the family of coordinate surfaces $x_{i}=$ const are given by

$$
I_{i}=H_{j}^{2} d x_{j}^{2}+H_{k}^{2} d x_{k}^{2}
$$

and therefore the two principal curvatures of these coordinate surfaces read

$$
\kappa_{i j}=-\frac{\beta_{i j}}{H_{j}} \quad \text { and } \quad \kappa_{i k}=-\frac{\beta_{i k}}{H_{k}} .
$$


Hence, due to Lamé's equations (8), the metric coefficient $\left.H_{i}\right|_{x_{i}=\text { const }}$ is a solution of the point equation (7) of the coordinate surface $x_{i}=$ const.

Remarkable subclasses of triply orthogonal systems emerge from induced metrics whose traces fulfill a certain condition (cf. [2,11,19]). We use the following terminology:

Definition 2.1. ([19, Sect. 64]) A $\chi$-system is a triply orthogonal system such that the trace with respect to the Minkowski-metric of the induced metric $I$ satisfies

$$
H_{1}^{2}+H_{2}^{2}-H_{3}^{3}=\chi .
$$

In particular, a 0 -system is called a Guichard net.

Another distinguished subclass in this realm consists of $\alpha^{2}|f|^{2}$-systems, where $\alpha \in \mathbb{R}$ (see [11, Chap.X]). As well as Guichard nets, this class is invariant under inversions: let $\left(f, H_{1}, H_{2}, H_{3}\right)$ be an $\alpha^{2}|f|^{2}$-system, then

$$
\begin{gathered}
f \rightarrow f^{\prime}:=\frac{f}{|f|^{2}} \\
H_{i}^{2} \rightarrow{H_{i}^{\prime}}^{2}:=\frac{H_{i}^{2}}{|f|^{4}}
\end{gathered}
$$

and, therefore, $f^{\prime}$ is an $\alpha^{2}\left|f^{\prime}\right|^{2}$-system.

Better insights into the geometry of a triply orthogonal system is often obtained by studying the coordinate surfaces. Thus, many geometric ideas known from surface theory were classically carried over to triply orthogonal systems by requiring a geometric property for any coordinate surface of the system: for example, systems consisting of isothermic surfaces or of constant Gaussian curvature surfaces. Also the rich transformation theory for triply orthogonal systems is based on this concept.

The Combescure transformation between two triply orthogonal systems is named after its first appearance in [9]:

Definition 2.2. Two triply orthogonal systems $f, \hat{f}: U \rightarrow \mathbb{R}^{3}$ are related by a Combescure transformation if any two corresponding coordinate surfaces of $f$ and $\hat{f}$ are Combescure transforms of each other.

Since the rotational coefficients $\beta_{i j}$ determine the normal directions of the coordinate surfaces of a triply orthogonal system, two systems are related by a Combescure transformation if and only if they share the same rotational coefficients (cf. [26, Sect. 5]).

The following proposition gives a criterion for three functions to define a Combescure transformation of a given parametrized triply orthogonal system:

Proposition 2.3. Let $f$ be a triply orthogonal system with Lamé coefficients $\left(H_{i}\right)_{i}$, then

$$
d \hat{f}=h_{1} \partial_{x} f d x+h_{2} \partial_{y} f d y+h_{3} \partial_{z} f d z
$$

defines a Combescure transform $\hat{f}$ of $f$ if and only if

$$
\partial_{i} h_{j}=\left(h_{i}-h_{j}\right) \partial_{i} \ln H_{j} .
$$


Proof. Let us denote the rotational coefficients of $\hat{f}$ by $\hat{\beta}_{i j}:=\frac{1}{h_{i} H_{i}} \partial_{i}\left(h_{j} H_{j}\right)$, then

$$
\beta_{i j}-\hat{\beta}_{i j}=\frac{H_{j}}{h_{i} H_{i}}\left\{\left(h_{i}-h_{j}\right) \partial_{i} \ln H_{j}-\partial_{i} h_{j}\right\} .
$$

Hence, $\beta_{i j}=\hat{\beta}_{i j}$ if and only if the equations (14) hold, which proves the claim.

Thus, for two given metrics $I=\sum_{i=1}^{3} H_{i}^{2} d x_{i}^{2}$ and $\hat{I}=\sum_{i=1}^{3}\left(h_{i} H_{i}\right)^{2} d x_{i}^{2}$, where the functions $h_{1}, h_{2}$ and $h_{3}$ satisfy equations (14), we can find two parametrizations $f$ and $\hat{f}$ which have these metrics as induced metrics and are related by a Combescure transformation. Therefore, we also say that two such induced metrics are related by a Combescure transformation.

To conclude this section, we prove a rather technical result, which will become important later. Suppose we start with a triply orthogonal system and Combescure transform each coordinate surface. Then the following proposition gives a criterion in which cases the Combescure transformed surfaces constitute again a triply orthogonal system:

Proposition 2.4. Let $\left(f, H_{1}, H_{2}, H_{3}\right)$ be a triply orthogonal system and let $\varphi_{1}, \varphi_{2}$ and $\varphi_{3}$ denote three functions that define by (5) Combescure transformations for the corresponding family of coordinate surfaces $x_{i}=$ const. Then, there exists a Combescure transformed triply orthogonal system $\hat{f}$ of $f$ such that the Combescure transformations between the coordinate surfaces are induced by the corresponding functions $\varphi_{1}, \varphi_{2}$ and $\varphi_{3}$ if and only if

$$
\varphi_{1}+\varphi_{2}+\varphi_{3}=0 \text { and } \partial_{j} \varphi_{j}=\varphi_{i} \partial_{j} \ln H_{k}+\varphi_{k} \partial_{j} \ln H_{i} .
$$

Proof. By Proposition 2.3, any Combescure transform $\hat{f}$ of $f$ can be described by three functions $h_{1}, h_{2}$ and $h_{3}$ satisfying (14). On the other hand, on the level of coordinate surfaces, any function $\varphi_{j}$ gives rise to functions $h_{i}^{j}$ and $h_{k}^{j}$ fulfilling [cf. conditions (5)]

$$
\begin{aligned}
& \partial_{k} h_{i}^{j}=\varphi_{j} \partial_{k} \ln H_{i} \\
& \partial_{i} h_{i}^{j}=-\varphi_{j} \partial_{i} \ln \left(H_{k} \varphi_{j}\right) \\
& \partial_{k} h_{k}^{j}=\varphi_{j} \partial_{k} \ln \left(H_{i} \varphi_{j}\right) \\
& \partial_{i} h_{k}^{j}=-\varphi_{j} \partial_{i} \ln H_{k} .
\end{aligned}
$$

Therefore, we obtain a Combescure transformed triply orthogonal system $\hat{f}$ of $f$ with the required property if and only if $h_{j}^{i}=h_{j}^{k}$.

Thus, if we assume that the conditions (15) are satisfied, then

$$
\partial_{j} h_{j}^{i}=\varphi_{i} \partial_{j} \ln \left(H_{k} \varphi_{i}\right)=-\varphi_{k} \partial_{j} \ln \left(H_{i} \varphi_{k}\right)=\partial_{j} h_{j}^{k}
$$

hold. Hence, it remains to show that the system

$$
\begin{aligned}
\partial_{j} h_{j} & =\varphi_{i} \partial_{j} \ln \left(H_{k} \varphi_{i}\right) \\
\partial_{k} h_{j} & =-\varphi_{i} \partial_{k} \ln H_{j} \\
\partial_{i} h_{j} & =\varphi_{k} \partial_{i} \ln H_{j}
\end{aligned}
$$


is indeed integrable. Since $\varphi_{i}$ and $\varphi_{k}$ induce Combescure transformations, we obtain by Eq. (5) the integrability conditions

$$
\partial_{j}\left(\partial_{k} h_{j}\right)=\partial_{k}\left(\partial_{j} h_{j}\right) \text { and } \partial_{i}\left(\partial_{j} h_{j}\right)=\partial_{j}\left(\partial_{i} h_{j}\right) .
$$

The remaining integrability condition $\partial_{i}\left(\partial_{k} h_{j}\right)=\partial_{k}\left(\partial_{i} h_{j}\right)$ then follows from the assumptions (15) and the first system of Lamé's equations of $f$.

Conversely, suppose that $h_{j}^{i}=h_{j}^{k}$, then Eq. (16) is satisfied and we conclude that the conditions (5) hold.

\section{G-surfaces and G-systems}

\subsection{G-surfaces}

We present, compared to the definition given by Calapso in [6, Sect. VI]), a slightly generalized definition of G-surfaces, where we allow for three different subclasses depending on the choice of $\varepsilon \in\{0, \pm 1\}$ :

Definition 3.1. A surface $f: M^{2} \rightarrow \mathbb{R}^{3}$ is called a $G$-surface if there exist curvature line coordinates with induced metric $I=H_{1}^{2} d x^{2}+H_{2}^{2} d y^{2}$ and a Combescure transform $\hat{f}$ of $f$ such that

$$
c H_{1}^{2} H_{2}^{2}\left(\frac{\kappa_{1}}{\hat{\kappa}_{1}}-\frac{\kappa_{2}}{\hat{\kappa}_{2}}\right)^{2}=H_{2}^{2}+\varepsilon H_{1}^{2}
$$

for some $\varepsilon \in\{0, \pm 1\}, c \in \mathbb{R} \backslash\{0\}$ and $\kappa_{1}, \kappa_{2}, \hat{\kappa}_{1}$ and $\hat{\kappa}_{2}$ the principal curvatures of $f$ and $\hat{f}$, respectively.

The surface $\hat{f}$ will be called an associated surface of $f$.

Obviously, the G-condition (18) depends on the choice of particular curvature line coordinates. However, from the definition we immediately deduce that a surface given in (arbitrary) curvature line coordinates $(x, y)$ is a G-surface if and only if there exist functions $\chi_{1}=\chi_{1}(x)$ and $\chi_{2}=\chi_{2}(y)$ such that

$$
c\left(\frac{\kappa_{1}}{\hat{\kappa}_{1}}-\frac{\kappa_{2}}{\hat{\kappa}_{2}}\right)^{2}=\frac{1}{\chi_{1}^{2} H_{1}^{2}}+\frac{\varepsilon}{\chi_{2}^{2} H_{2}^{2}} .
$$

As already observed in [6, Sect. VI (89)]), a G-surface admits a special solution of its point equation:

Proposition 3.2. A surface $f: M^{2} \rightarrow \mathbb{R}^{3}$ is a $G$-surface if and only if there exist curvature line coordinates such that the function $\sqrt{H_{2}^{2}+\varepsilon H_{1}^{2}}$ is a scalar solution of its point Eq. (1).

Proof. By definition a surface is a G-surface if and only if there exist curvature line coordinates and a Combescure transformation determined by functions $h$ and $l$ such that

$$
(h-l)^{2}=\frac{H_{2}^{2}+\varepsilon H_{1}^{2}}{c H_{1}^{2} H_{2}^{2}} .
$$


Hence, this is the case if and only if the function $\varphi:=\frac{\sqrt{H_{2}^{2}+\varepsilon H_{1}^{2}}}{\sqrt{c} H_{1} H_{2}}$ fulfills Eq. (4). A straightforward computation shows that Eq. (4) holds if and only if $\sqrt{H_{2}^{2}+\varepsilon H_{1}^{2}}$ is a solution of the point equation of $f$.

As a consequence of the construction in this proof, the function $\varphi$ gives rise to a 1-parameter family of Combescure transformations of $f$ such that the G-condition (18) is satisfied. Hence, any G-surface admits (up to orientation) a 1-parameter family of associated surfaces: let $\hat{f}_{0}$ be an arbitrary associated surface of $f$, then this 1 -parameter family $\left(\hat{f}_{c}\right)_{c \in \mathbb{R}}$ is given by

$$
\hat{f}_{c}:=\hat{f}_{0}+c f \text {. }
$$

In particular, any three associated surfaces $\hat{f}_{1}, \hat{f}_{2}$ and $\hat{f}_{3}$ satisfy the relation

$$
\left(\hat{f_{1}}-\hat{f_{3}}\right)=\lambda\left(\hat{f_{2}}-\hat{f_{3}}\right)
$$

for a constant $\lambda \in \mathbb{R} \backslash\{0\}$.

As many integrable surface classes, we will prove that also G-surfaces can be characterized by the existence of Combescure transforms fulfilling a special relation between their principal curvatures:

Theorem 3.3. A surface $f$ is a G-surface if and only if there exists a non-trivial pair $\left(\hat{f}, f^{\star}\right)$ of Combescure transforms of $f$ with principal curvatures satisfying

$$
\frac{1}{\kappa_{1} \kappa_{2}^{\star}}+\frac{1}{\kappa_{2} \kappa_{1}^{\star}}=-\frac{2}{\hat{\kappa}_{1} \hat{\kappa}_{2}} \text {. }
$$

A Combescure related pair $\left(\hat{f}, f^{\star}\right)$ of $f$ will be called non-trivial if

$$
\kappa_{1} \kappa_{1}^{\star} \neq-\hat{\kappa}_{1}^{2} \text { or } \kappa_{2} \kappa_{2}^{\star} \neq-\hat{\kappa}_{2}^{2} .
$$

Remark 3.4. Note that any arbitrary surface $f$ admits a pair of trivial Combescure transforms such that the relation (20) holds: namely, for any $\lambda \in \mathbb{R} \backslash\{0\}$, the choice

$$
\hat{f}:=\lambda f \quad \text { and } \quad f^{\star}:=-\lambda^{2} f
$$

provides two such Combescure transforms of $f$.

Proof of Theorem 3.3. Suppose that $f$ is a G-surface with induced metric

$$
I=H_{1}^{2} d x^{2}+H_{2}^{2} d y^{2}
$$

and let $\hat{f}$ be an associated surface defined by

$$
\partial_{x} \hat{f}=h \partial_{x} f \text { and } \partial_{y} \hat{f}=l \partial_{y} f
$$

with principal curvatures $\hat{\kappa}_{1}$ and $\hat{\kappa}_{2}$. 
We will prove that the sought-after Combescure transform $f^{\star}$ of $f$ is, for a suitable choice of $\tilde{\delta} \in\{ \pm 1\}$, obtained by integrating

$$
d f^{\star}=\tilde{\delta}\left(-h^{2}+\frac{1}{H_{1}^{2}}\right) \partial_{x} f d x+\tilde{\delta}\left\{-l^{2}+\frac{\varepsilon}{H_{2}^{2}}\right\} \partial_{y} f d y .
$$

Indeed, a straightforward computation using the G-condition (18) shows that (22) is integrable. Since the principal curvatures of $f^{\star}$ are given by

$$
\kappa_{1}^{\star}=\tilde{\delta} \delta \frac{H_{1}^{2} \kappa_{1}}{-h^{2} H_{1}^{2}+1} \text { and } \kappa_{2}^{\star}=\tilde{\delta} \delta \frac{H_{2}^{2} \kappa_{2}}{-l^{2} H_{2}^{2}+\varepsilon},
$$

where $\delta \in\{ \pm 1\}$ depends on the orientation of the normal of $f^{\star}$, we obtain that

$$
\tilde{\delta} \delta\left(\frac{1}{\kappa_{1} \kappa_{2}^{\star}}+\frac{1}{\kappa_{2} \kappa_{1}^{\star}}\right)=-\frac{2 h l}{\kappa_{1} \kappa_{2}}=-\frac{2}{\hat{\kappa}_{1} \hat{\kappa}_{2}} .
$$

Hence, for the choice $\tilde{\delta}=\delta$, we have constructed the sought-after pair $\left(\hat{f}, f^{\star}\right)$, which is non-trivial.

Conversely, suppose that $\hat{f}$ and $f^{\star}$ are non-trivial Combescure transforms of $f$ which satisfy condition (20). Then, there exist functions $h^{\prime}, l^{\prime}$ and $h^{\prime \prime}, l^{\prime \prime}$ such that

$$
\begin{array}{ll}
H_{1}=h^{\prime} \hat{H}_{1}, & H_{2}=l^{\prime} \hat{H}_{2}, \\
H_{1}^{\star}=h^{\prime \prime} \hat{H}_{1}, & H_{2}^{\star}=l^{\prime \prime} \hat{H}_{2}
\end{array}
$$

and the principal curvatures of $f$ and $f^{\star} \operatorname{read} \kappa_{1}=\hat{\kappa}_{1} / h^{\prime}$ and $\kappa_{1}^{\star}=\hat{\kappa}_{1} / h^{\prime \prime}$, respectively. From Eq. (20), we then deduce that

$$
h^{\prime} l^{\prime \prime}+l^{\prime} h^{\prime \prime}=-2
$$

holds.

Moreover, from the compatibility conditions (3) and (23) we obtain

$$
\begin{gathered}
\partial_{y}\left(h^{\prime} h^{\prime \prime}\right)=-2\left\{1+h^{\prime} h^{\prime \prime}\right\} \partial_{y} \ln \hat{H}_{1}, \\
\partial_{x}\left(l^{\prime} l^{\prime \prime}\right)=-2\left\{1+l^{\prime} l^{\prime \prime}\right\} \partial_{x} \ln \hat{H}_{2}
\end{gathered}
$$

and therefore

$$
\begin{aligned}
h^{\prime} h^{\prime \prime} & =-1+\frac{\psi_{1}(x)}{\hat{H}_{1}^{2}}, \\
l^{\prime} l^{\prime \prime} & =-1+\frac{\psi_{2}(y)}{\hat{H}_{2}^{2}}
\end{aligned}
$$

for suitable functions $\psi_{1}(x)$ and $\psi_{2}(y)$. Since, by (21), we have

$$
h^{\prime} h^{\prime \prime} \neq 1 \text { or } l^{\prime} l^{\prime \prime} \neq 1
$$

at least one of the functions $\psi_{1}(x)$ and $\psi_{2}(y)$ does not vanish. 
Furthermore, substituting in Eq. (23), gives

$$
\left(\frac{\kappa_{1}}{\hat{\kappa}_{1}}-\frac{\kappa_{2}}{\hat{\kappa}_{2}}\right)^{2}=\frac{\psi_{2}(y)}{H_{2}^{2}}+\frac{\psi_{1}(x)}{H_{1}^{2}},
$$

which leads, for a suitable choice of $\varepsilon \in\{0, \pm 1\}$ and $c \in \mathbb{R} \backslash\{0\}$ to the G-condition (19). Thus, $f$ is a G-surface.

Therefore, due to symmetry, it follows that $\hat{f}$ is also an associated surface of $f^{\star}$ and we obtain another G-surface:

Corollary and Definition 3.5. Let $f$ be a $G$-surface, then the Combescure transform $f^{\star}$ defined by (20) is a G-surface, which will be called the dual surface of $f$ with respect to the associated surface $\hat{f}$.

Note that a dual surface depends on the choice of the associated surface (Figs. 1, 2): let $\hat{f}_{0}$ be an associated surface with corresponding dual surface $f_{0}^{\star}$, then the dual surface $f_{c}^{\star}$ with respect to the associated surface $\hat{f}_{c}$ is given by

$$
\begin{aligned}
& f_{c}^{\star}:=f_{0}^{\star}-2 c \hat{f}_{0}-c^{2} f=f_{0}^{\star}-2 c \hat{f}_{\frac{c}{2}} .
\end{aligned}
$$

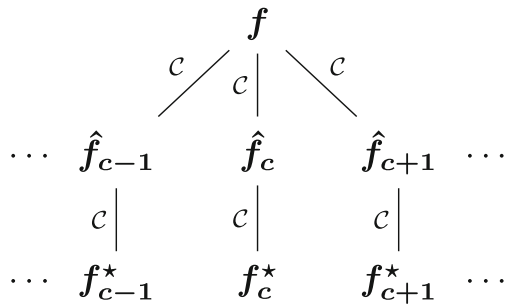

Fig. 1. A G-surface $f$ with a 1-parameter family of Combescure related associated surfaces $\left(\hat{f}_{c}\right)_{c \in \mathbb{R}}$ and the corresponding dual surfaces $\left(f_{c}^{\star}\right)_{c \in \mathbb{R}}$
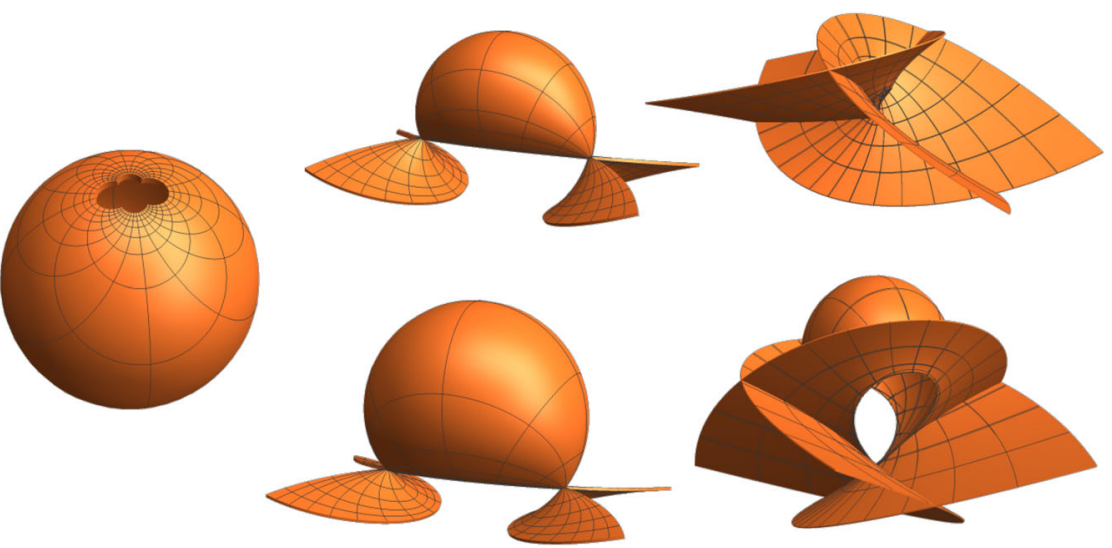

Fig. 2. A sphere with two Dupin cyclides as associated surfaces (middle) and the two corresponding dual surfaces (right) 
Furthermore, let us fix some dual surface $f_{\bar{c}}^{\star}$ of $f$. Then the 1-parameter family of associated surfaces of $f_{\bar{c}}^{\star}$ becomes

$$
\hat{f}_{\bar{c} d}:=\hat{f}_{\bar{c}}+d f_{\bar{c}}^{\star}, \quad d \in \mathbb{R}
$$

while the corresponding dual surfaces of $f_{\bar{c}}^{\star}$ are given by

$$
\left(f_{\bar{c}}^{\star}\right)_{d}^{\star}:=f-2 d \hat{f}_{\bar{c}}-d^{2} f_{\bar{c}}^{\star} .
$$

Hence, we recover the original surface $f$ in the dual family of any dual surface $f_{\bar{c}}^{\star}$ of $f$ at $d=0$,

$$
\left(f_{\bar{c}}^{\star}\right)_{0}^{\star}=f
$$

and the described construction indeed provides a duality.

We further remark that, generically, the two associated families $\left(\hat{f}_{c}\right)_{c \in \mathbb{R}}$ and $\left(\hat{f}_{\bar{c} d}\right)_{d \in \mathbb{R}}$ do not coincide, while for $d \neq 0$ the dual surfaces $\left(f_{\bar{c}}^{\star}\right)_{d \in \mathbb{R}}^{\star}$ amount to scalings of the family $\left(f_{c}^{\star}\right)_{c \in \mathbb{R}}(\mathrm{cf}$. Example 4.1 in Sect. 4$)$.

Apart from providing new G-surfaces from a given one, the characterization discussed in Theorem 3.3 shows that G-surfaces are contained in the integrable class of O-surfaces [27]: if we consider a non-trivial triplet of Combescure transformations and equip the dual space with the indefinite metric induced by the matrix

$$
\left(\begin{array}{lll}
0 & 1 & 0 \\
1 & 0 & 0 \\
0 & 0 & 2
\end{array}\right),
$$

then the orthogonality condition that defines the corresponding subclass of Osurfaces, is equivalent to the condition (20).

However, note that the requirement of non-trivial Combesure transforms is indeed necessary: by Remark 3.4, any surface satisfies the criterion of an O-surface with respect to the indefinite metric induced by (24), if trivial Combescure transformations are not excluded.

\subsection{Examples}

Using the just derived characterizations we demonstrate how various well-known surface classes arise in the context of G-surfaces.

Isothermic surfaces. Since any isothermic surface admits conformal curvature line coordinates, any scaling of $f$ provides an associated surface as seen from the definition using $\varepsilon=-1$. The converse also holds:

Proposition 3.6. A G-surface $f$ is isothermic if and only if the family of associated surfaces $\hat{f}_{c}$ is given by scalings of $f$, that is, $\hat{f}_{c}=c f$ for all $c \in \mathbb{R}$. 
As a consequence, in the context of G-surfaces, the dual surfaces of an isothermic surface $f$ with induced metric $I=e^{2 \psi}\left\{d x^{2}+d y^{2}\right\}$ are determined by

$$
d f_{c}^{\star}=\left\{-c^{2}+e^{-2 \psi}\right\} \partial_{x} f d x+\left\{-c^{2}-e^{-2 \psi}\right\} \partial_{y} f d y .
$$

Hence, if for $c=0$ the associated surface degenerates to a point, the corresponding dual surface becomes the classical Christoffel dual [8] of an isothermic surface and therefore it is again isothermic. However, the other dual surfaces $f_{c}^{\star}$ are in general not isothermic again.

Guichard surfaces. Recall [5] that a surface is a Guichard surface if and only if it fulfills Calapso's equation

$$
c\left(\kappa_{1}-\kappa_{2}\right)^{2} H_{1}^{2} H_{2}^{2}=H_{2}^{2}+\varepsilon H_{1}^{2}
$$

for suitable curvature line coordinates, $\varepsilon \in\{ \pm 1,0\}$ and some $c \in \mathbb{R} \backslash\{0\}$.

Hence, since the spherical representative of a surface is a Combescure transform that is determined by the functions $h=\kappa_{1}$ and $l=\kappa_{2}$, any Guichard surface is a G-surface by Calapso's equation.

Moreover, the spherical representative as an associated surface distinguishes this subclass:

Proposition 3.7. A G-surface $f$ is a Guichard surface if and only if its spherical representative is an associated surface of $f$.

We remark that, in the case of a Guichard surface, condition (20) becomes the relation originally used to define Guichard surfaces [18], namely

$$
\frac{1}{\kappa_{1} \kappa_{2}^{\star}}+\frac{1}{\kappa_{2} \kappa_{1}^{\star}}=-2
$$

Therefore, the dual surface $f^{\star}$ with respect to the spherical representative, constructed in the context of G-surfaces, coincides with the classical dual surface given by Guichard.

This subclass also provides examples of G-surfaces that satisfy the G-condition for $\varepsilon=0$ : in this case, we deduce from the G-condition and the compatibility conditions (3) that the associated surfaces are determined by functions $h$ and $l$ of the form

$$
\begin{aligned}
& h= \pm \frac{1}{H_{1}^{2}}+\psi_{1} \\
& l=\psi_{1},
\end{aligned}
$$

where $\psi_{1}=\psi_{1}(x)$.

Thus, for a Guichard surface, where one of the associated surfaces is given by the spherical representative, one of the principal curvatures of $f$ is constant along the corresponding curvature direction. Therefore, $f$ is a channel surface (cf. [5]). 
$\Omega$-surfaces. By Demoulin's equation [12], a surface is an $\Omega$-surface if and only if there exists a Combescure transformed dual surface $f^{\star}$ fulfilling

$$
\left(\frac{1}{\kappa_{1}}-\frac{1}{\kappa_{2}}\right)\left(\frac{1}{\kappa_{1}^{\star}}-\frac{1}{\kappa_{2}^{\star}}\right)=\frac{1}{\kappa_{1}^{2} H_{1}^{2}}+\frac{\varepsilon}{\kappa_{2}^{2} H_{2}^{2}},
$$

for a suitable choice of curvature line coordinates inducing the first fundamental form $I=H_{1}^{2} d x^{2}+H_{2}^{2} d y^{2}$.

A reformulation of the condition (20) between the principal curvatures of a Gsurface and its associated and dual surfaces, will shed light on the relation between the dual surfaces of a G-surface and of an $\Omega$-surface:

Lemma 3.8. Let $f$ be a $G$-surface, then the principal curvatures of an associated surface $\hat{f}$ and the corresponding dual surface $f^{\star}$ satisfy

$$
\left(\frac{1}{\kappa_{1}}-\frac{1}{\kappa_{2}}\right)\left(\frac{1}{\kappa_{1}^{\star}}-\frac{1}{\kappa_{2}^{\star}}\right)=\frac{1}{\kappa_{1}^{2} H_{1}^{2}}+\frac{\varepsilon}{\kappa_{2}^{2} H_{2}^{2}}-\left(\frac{1}{\hat{\kappa}_{1}}-\frac{1}{\hat{\kappa}_{2}}\right)^{2} .
$$

Proof. Suppose $f$ is a G-surface. Then the principal curvatures of a dual surface with respect to the associated surface

$$
d \hat{f}=h \partial_{x} f d x+l \partial_{y} f d y
$$

are given by

$$
\begin{aligned}
\kappa_{1}^{\star} & =\frac{\kappa_{1} H_{1}^{2}}{-h^{2} H_{1}^{2}+1}, \\
\kappa_{2}^{\star} & =\frac{\kappa_{2} H_{2}^{2}}{-l^{2} H_{2}^{2}+\varepsilon} .
\end{aligned}
$$

Now a straightforward computation using the G-condition (18) proves the claim.

Therefore, a dual surface of a G-surface fulfills Demoulin's equation (27) if and only if

$$
l \kappa_{1}-h \kappa_{2}=0 .
$$

Thus, we have proven:

Proposition 3.9. The dual surface of a $G$-surface provides an $\Omega$-dual surface if and only if the corresponding associated surface is totally umbilic or degenerates to a point.

In particular, this proposition reflects that isothermic and Guichard surfaces belong to the classes of $G$-as well as that of $\Omega$-surfaces. In these cases, the corresponding associated surface coincides with the associated Gauss map for $\Omega$-surfaces, which was recently determined in [25]. 


\subsection{G-systems}

Although it is quite popular to distinguish surface classes by the existence of particular Combescure transforms, there are only few results in this direction in the realm of triply orthogonal systems (for example, the Combescure transforms of the confocal quadrics [26]).

However, it is a prominent question to analyse systems consisting of particular coordinate surfaces: for example, systems with isothermic coordinate surfaces [11, Chap. III-V] or Bianchi's systems consisting of coordinate surfaces with constant Gaussian curvature (cf. [22,26]). Clearly, these systems are all built from G-surfaces (cf. Propositions 3.6 and 3.7).

Here, we are interested in both aspects and investigate triply orthogonal systems consisting of G-surfaces that admit Combescure transforms constituted by the associated and dual surfaces. In particular, it will turn out that Guichard nets belong to this class of triply orthogonal systems.

Definition 3.10. A triply orthogonal system $f$ that admits non-trivial Combescure transforms $\hat{f}$ and $f^{\star}$ such that the principal curvatures of the coordinate surfaces satisfy

$$
\frac{1}{\kappa_{i j} \kappa_{i k}^{\star}}+\frac{1}{\kappa_{i k} \kappa_{i j}^{\star}}=-\frac{2}{\hat{\kappa}_{i j} \hat{\kappa}_{i k}}
$$

will be called a $G$-system. The Combescure transforms $\hat{f}$ and $f^{\star}$ are said to be an associated system and a dual system, respectively.

If we describe the Combescure transform $\hat{f}$ of a G-system $f$ by

$$
d \hat{f}=h_{1} \partial_{x} f d x+h_{2} \partial_{y} f d y+h_{3} \partial_{z} f d z,
$$

we deduce, using similar arguments as in the proof of Theorem 3.3 that there exist functions $\psi_{i}=\psi_{i}\left(x_{i}\right)$ such that

$$
\left(h_{j}-h_{k}\right)^{2}=\frac{1}{\psi_{k} H_{k}^{2}}+\frac{1}{\psi_{j} H_{j}^{2}} .
$$

Hence, the coordinate surfaces of a G-system are G-surfaces and $\hat{f}$ consists of the corresponding associated surfaces.

The corresponding dual system $f^{\star}$ is then given by

$$
d f^{\star}=h_{1}^{\star} \partial_{x} f d x+h_{2}^{\star} \partial_{y} f d y+h_{3}^{\star} \partial_{z} f d z, \text { where } h_{i}^{\star}=-h_{i}^{2}+\frac{1}{\psi_{i} H_{i}^{2}}
$$

and therefore the coordinate surfaces of $f^{\star}$ are dual surfaces of the coordinate surfaces of $f$. In particular, the existence of a dual system $f^{\star}$ just follows from the existence of the associated system $\hat{f}$.

In summary, we have proven

Proposition 3.11. The coordinate surfaces of a $G$-system are $G$-surfaces and the Combescure transforms $\hat{f}$ and $f^{\star}$ satisfying (28) consist of the associated and dual surfaces of the coordinate surfaces of $f$, respectively. 


\section{Guichard nets}

Recall from Sect. 2.2 that a Guichard net $\left(f, H_{1}, H_{2}, H_{3}\right)$ is a 0 -system; hence, by setting $\left(\varepsilon_{1}, \varepsilon_{2}, \varepsilon_{3}\right)=(1,1,-1)$, these systems satisfy the Guichard condition

$$
\varepsilon_{1} H_{1}^{2}+\varepsilon_{2} H_{2}^{2}+\varepsilon_{3} H_{3}^{2}=0
$$

and, by differentiating, the following relation

$$
\varepsilon_{i} \partial_{i} H_{i}+\varepsilon_{j} H_{j} \beta_{i j}+\varepsilon_{k} H_{k} \beta_{i k}=0
$$

between the Lamé coefficients and the rotational coefficients.

Theorem 4.1. A Guichard net is a G-system and, in particular, the coordinate surfaces of a Guichard net are G-surfaces.

Proof. Let $\left(f, H_{1}, H_{2}, H_{3}\right)$ be a Guichard net. From Lamé's first system (8) and the Guichard condition we learn that the functions

$$
\pm \sqrt{H_{1}^{2}+H_{2}^{2}}, \pm \sqrt{H_{3}^{2}-H_{1}^{2}} \text { and } \pm \sqrt{H_{3}^{2}-H_{2}^{2}}
$$

are solutions of the point equation of the corresponding coordinate surface family. Thus, by Proposition 3.2, the coordinate surfaces of a Guichard net are G-surfaces and the functions

$$
\varphi_{3}:=\frac{H_{3}}{H_{1} H_{2}}, \quad \varphi_{2}:=-\frac{H_{2}}{H_{1} H_{3}}, \quad \varphi_{1}:=-\frac{H_{1}}{H_{2} H_{3}}
$$

induce the corresponding Combescure transformations that transform any coordinate surface to the associated surfaces.

Moreover, Proposition 2.4 guarantees that, if suitably chosen, the associated surfaces constitute again a triply orthogonal system: by the Guichard condition and (32), we obtain

$$
\begin{aligned}
& \varphi_{1}+\varphi_{2}+\varphi_{3}=0, \\
& \partial_{j} \varphi_{j}-\varphi_{i} \partial_{j} \ln H_{k}-\varphi_{k} \partial_{j} \ln H_{i} \\
& \quad=\frac{1}{H_{i} H_{j} H_{k}}\left\{\varepsilon_{j} H_{j} \partial_{j} H_{j}+\varepsilon_{k} H_{k} \partial_{j} H_{k}+\varepsilon_{i} H_{i} \partial_{j} H_{i}\right\}=0
\end{aligned}
$$

and therefore the functions $\varphi_{1}, \varphi_{2}$ and $\varphi_{3}$ induce a 1-parameter family of Combescure transformed systems. By construction, these are the associated systems of the Guichard net $f$.

Thus, Eq. (30) describes the corresponding dual systems of $f$ and a Guichard net is indeed a G-system.

To gain more insights into the geometry of Guichard nets, we explicitly construct its associated systems: 
Proposition 4.2. Let $f$ be a Guichard net, then the associated systems $\hat{f}$ of $f$ are 1-systems given by

$$
d \hat{f}=h_{1} \partial_{x} f d x+h_{2} \partial_{y} f d y+h_{3} \partial_{z} f d z
$$

where the functions $h_{1}, h_{2}$ and $h_{3}$ are determined by

$$
\begin{aligned}
\partial_{x} h_{3} & =-\frac{H_{2}}{H_{3}} \kappa_{13}, \\
\partial_{y} h_{3} & =\frac{H_{1}}{H_{3}} \kappa_{23}, \\
\partial_{z} h_{3} & =-\frac{H_{1} H_{2}}{H_{3}^{2}}\left(\kappa_{31}-\kappa_{32}\right), \\
h_{1} & =h_{3}+\frac{H_{2}}{H_{1} H_{3}}, \\
h_{2} & =h_{3}-\frac{H_{1}}{H_{2} H_{3}} .
\end{aligned}
$$

Proof. Suppose that $\left(f, H_{1}, H_{2}, H_{3}\right)$ is a Guichard net. From the proof of Theorem 4.1, we know that $f$ is a G-system with associated systems induced by the functions

$$
\varphi_{3}:=\frac{H_{3}}{H_{1} H_{2}}, \quad \varphi_{2}:=-\frac{H_{2}}{H_{1} H_{3}}, \quad \varphi_{1}:=-\frac{H_{1}}{H_{2} H_{3}} .
$$

Hence, from the system (17) in the proof of Proposition 2.4 and the Guichard conditions (31) and (32), we obtain the asserted system (34).

Moreover, these constructed associated systems are 1-systems, since

$$
\hat{H}_{1}^{2}+\hat{H}_{2}^{2}-\hat{H}_{3}^{2}=\left(h_{1}^{2}-h_{3}^{2}\right) H_{1}^{2}+\left(h_{2}^{2}-h_{3}^{2}\right) H_{2}^{2}=1 \text {. }
$$

Conversely, from the existence of a particular Combescure transformed triply orthogonal system, we can conclude that the Guichard condition is satisfied.

Thus, we obtain a characterization of Guichard nets:

Theorem 4.3. A triply orthogonal system $\left(f, H_{1}, H_{2}, H_{3}\right)$ is a Guichard net if and only if there exists a Combescure transformed system $\left(\hat{f}, \hat{H}_{1}, \hat{H}_{2}, \hat{H}_{3}\right)$ such that the relations

$$
H_{i} \hat{H}_{j}-H_{j} \hat{H}_{i}=\varepsilon_{k} H_{k}
$$

between the Lamé coefficients of $f$ and $\hat{f}$ hold. In this case, the system $\hat{f}$ is an associated system of the Guichard net. 
Proof. Suppose that $\hat{f}$ is a Combescure transform of $f$ fulfilling (35) and define $h_{i}:=\frac{\hat{H}_{i}}{H_{i}}$. Then we obtain

$$
\frac{1}{H_{1} H_{2} H_{3}}\left\{H_{1}^{2}+H_{2}^{2}-H_{3}^{2}\right\}=h_{3}-h_{2}+h_{1}-h_{3}-h_{1}+h_{2}=0
$$

and $f$ is indeed a Guichard net.

Conversely, let $f$ be a Guichard net. Then, by Proposition 4.2, there exist associated systems given by (34) which fulfill

$$
h_{j}-h_{i}=\frac{\varepsilon_{k} H_{k}}{H_{i} H_{j}} .
$$

Hence, any associated system yields a sought-after Combescure transform and the claim is proven.

From Proposition 4.2, we obtain, similar to the case of G-surfaces, the following relation between the associated systems: if $\hat{f}_{0}$ is an associated system induced by the functions $h_{1}, h_{2}$ and $h_{3}$ satisfying system (34), then the 1-parameter family $\hat{f}_{c}$ of associated systems are induced by the functions $h_{i c}:=h_{i}+c$, where $c \in \mathbb{R}$. Hence, we obtain

$$
\hat{f}_{c}=\hat{f_{0}}+c f .
$$

As a consequence, we conclude that the dual systems of a Guichard net are given by

$$
d f_{c}^{\star}=h_{1 c}^{\star} \partial_{x} f d x+h_{2 c}^{\star} \partial_{y} f d y+h_{3 c}^{\star} \partial_{z} f d z, \text { where } h_{i c}^{\star}=-\left(h_{i}+c\right)^{2}+\frac{\varepsilon_{i}}{H_{i}^{2}}(36)
$$

The characterization of Guichard nets given in Theorem 4.3 then shows, that the dual systems provide a 1-parameter family of (generically) new Guichard nets:

Proposition 4.4. The dual systems of a Guichard net are again Guichard nets.

Proof. Let $f$ be a Guichard net with an associated system $\hat{f}$ and the corresponding dual system $f^{\star}$. We will show that

$$
H_{i}^{\star} \hat{H}_{j}-H_{j}^{\star} \hat{H}_{i}=-\varepsilon_{k} H_{k}^{\star},
$$

hence, a Combescure transformation defined by the functions $-h_{1},-h_{2}$ and $-h_{3}$ satisfies the conditions (35) of Theorem 4.3 and therefore the dual system $f^{\star}$ is a Guichard net.

To prove this relation, we use $\varepsilon_{i} \varepsilon_{j}=-\varepsilon_{k}$, formula (36) and the relations

$$
h_{i}=h_{k}+\frac{\varepsilon_{j} H_{j}}{H_{i} H_{k}} \text { and } h_{j}=h_{k}-\frac{\varepsilon_{i} H_{i}}{H_{j} H_{k}} \text {. }
$$

Then we obtain

$$
\begin{aligned}
H_{i}^{\star} \hat{H}_{j}-H_{j}^{\star} \hat{H}_{i} & =h_{i} h_{j}\left\{-H_{i} \hat{H}_{j}-H_{j} \hat{H}_{i}\right\}+\frac{1}{H_{i} H_{j}}\left\{\varepsilon_{i} h_{j} H_{j}^{2}-\varepsilon_{j} h_{i} H_{i}^{2}\right\} \\
& =\varepsilon_{k} h_{k}^{2} H_{k}-\frac{1}{H_{k}}=-\varepsilon_{k} H_{k}^{\star},
\end{aligned}
$$

which completes the proof. 
Remark 4.5. The special triply orthogonal systems discussed in this section arise as principal coordinate systems of particular hypersurfaces: Guichard nets give rise to 3-dimensional conformally flat hypersurfaces, while $\chi$-systems with $\chi \in \mathbb{R}$ constant appear in the realm of hypersurfaces of a space form that can also be isometrically immersed as a hypersurface of another space form of a distinct constant curvature (cf. [7,20]). However, the relation between the geometry of the coordinate systems and the corresponding hypersurfaces is intricate and yet not fully examined.

For example, the duality of Guichard nets discussed in this work seems not directly related to the duality of conformally flat hypersurfaces given in [23]: while the dual hypersurface of the latter is constructed by using the Schouten tensor and is therefore (up to scaling) unique, any Guichard net admits a 1-parameter family of dual Guichard nets depending on the choice of the associated system. This ambiguity seems to be an interesting starting point for future work.

\subsection{Guichard nets with cyclic associated systems}

A triply orthogonal system is cyclic if two of the coordinate surface families consist of channel surfaces.

In this subsection we employ the geometric consequences for a Guichard net if all its associated systems are cyclic. It will turn out that these Guichard nets are rather special and contain a family of parallel or totally umbilic coordinate surfaces.

To begin, we recall some properties of such systems (cf. [16,26]) and remark some immediate relations to their associated systems.

If the coordinate surfaces of a Lamé family are parallel, then the other two families are developable surfaces formed by the normal lines along the curvature lines of the parallel surface family. Thus, two of the rotational coefficients vanish. Conversely, if for example $\beta_{j i}=\beta_{k i}=0$, then we conclude from (10) and (11) that the coordinate surfaces $x_{i}=$ const are parallel.

Consequently, a triply orthogonal system contains a family of parallel coordinate surfaces if and only if the corresponding coordinate surfaces of a (hence all) Combescure transforms are also parallel.

Moreover, taking into account construction (34) for the associated systems, we deduce two characterizations for the Guichard nets under consideration.

In the following paragraphs, the functions $h_{1}, h_{2}$ and $h_{3}$ fulfill the system (34) and therefore induce an associated system of the Guichard net.

Corollary 4.6. Let $f$ be a Guichard net with associated system $\hat{f}$. Then the following are equivalent:

(i) The coordinate surfaces $x_{i}=$ const of $f$ are parallel.

(ii) The coordinate surfaces $x_{i}=$ const of $\hat{f}$ are parallel.

(iii) $\partial_{j} h_{i}=\partial_{k} h_{i}=0$.

Corollary 4.7. The coordinate surfaces $x_{i}=$ const of a Guichard net are totally umbilic if and only if $\partial_{i} h_{i}=0$. 
These two characterizations in terms of the functions $h_{1}, h_{2}$ and $h_{3}$ enable us to understand Guichard nets with cyclic associated systems:

Proposition 4.8. A Guichard net contains a family of parallel or totally umbilic coordinate surfaces if and only if all its associated systems are cyclic.

Proof. Recall that a triply orthogonal system is $x_{i}$-cyclic, where the $x_{i}$-trajectories provide the circular direction, if and only if

$$
0=\partial_{i} \kappa_{j i}=\partial_{i} \kappa_{k i} \quad \Leftrightarrow 0=H_{i} \partial_{i} \beta_{j i}-\beta_{j i} \partial_{i} H_{i}=H_{i} \partial_{i} \beta_{k i}-\beta_{k i} \partial_{i} H_{i} .
$$

Thus, suppose that the associated systems are $x_{i}$-cyclic and denote the Lamé coefficients of them by $\hat{H}_{i}^{c}=\left(h_{i}+c\right) H_{i}$, where $c \in \mathbb{R}$. Then, we obtain

$$
\begin{gathered}
\text { for } c=0: h_{i} H_{i} \partial_{i} \beta_{j i}-\beta_{j i} \partial_{i}\left(h_{i} H_{i}\right)=h_{i} H_{i} \partial_{i} \beta_{k i}-\beta_{k i} \partial_{i}\left(h_{i} H_{i}\right)=0 \text { and } \\
\text { for } c \neq 0: h_{i} H_{i} \partial_{i} \beta_{j i}-\beta_{j i} \partial_{i}\left(h_{i} H_{i}\right)+c H_{i} \partial_{i} \beta_{j i}-c \beta_{j i} \partial_{i} H_{i} \\
=h_{i} H_{i} \partial_{i} \beta_{k i}-\beta_{k i} \partial_{i}\left(h_{i} H_{i}\right)+c H_{i} \partial_{i} \beta_{k i}-c \beta_{k i} \partial_{i} H_{i}=0,
\end{gathered}
$$

which shows that the corresponding Guichard net is $x_{i}$-cyclic. Therefore, we deduce from the circularity of the cyclic associated systems that

$$
\begin{aligned}
& 0=\partial_{i} \kappa_{j i}^{c \star}=\partial_{i} \frac{\kappa_{j i}}{h_{i}+c}, \\
& 0=\partial_{i} \kappa_{k i}^{c \star}=\partial_{i} \frac{\kappa_{k i}}{h_{i}+c} .
\end{aligned}
$$

As a consequence of the Corollaries 4.6 and 4.7, the coordinate surfaces $x_{i}=$ const are parallel (if $\kappa_{j i}=\kappa_{k i}=0$ ) or totally umbilic (if $\partial_{i} h_{i}=0$ ).

Conversely, assume that the Guichard net has a family of parallel coordinate surfaces, then, by Corollary 4.6, the corresponding coordinate surfaces of the associated systems are also parallel and therefore the associated systems are cyclic.

In the other case, if the coordinate surfaces $x_{i}=$ const of the Guichard net are totally umbilic, then the Guichard net is a cyclic system (cf. [20, Sect. 4]) and, by Corollary 4.7, we obtain $h_{i}=h_{i}\left(x_{j}, x_{k}\right)$. Thus, the principal curvatures $\kappa_{j i}^{\star}$ and $\kappa_{k i}^{\star}$ do not depend on $x_{i}$ and therefore the associated systems are $x_{i}$-cyclic.

Therefore, the associated systems of a Guichard net are triply orthogonal systems consisting of Dupin cyclides if and only if all coordinate surface families of the Guichard net are totally umbilic or parallel.

This leads to an interesting example which shows that, in general, the associated systems do not admit a reparametrization into a Guichard net.

Example 4.1. The so-called 6-sphere coordinates, obtained as inversion of the Cartesian coordinates and consisting of spheres that are all tangent to a fixed point, form a Guichard net. It is determined by the induced metric

$$
I=\frac{1}{\left(x^{2}+y^{2}+2 z^{2}\right)^{2}}\left\{d x^{2}+d y^{2}+2 d z^{2}\right\} .
$$


By solving system (34), we conclude that the induced metrics of the associated systems become

$$
\begin{aligned}
\hat{I}_{c}= & \left\{\frac{c+\sqrt{2}\left(y^{2}+z^{2}\right)}{x^{2}+y^{2}+2 z^{2}}\right\}^{2} d x^{2}+\left\{\frac{c-\sqrt{2}\left(x^{2}+z^{2}\right)}{x^{2}+y^{2}+2 z^{2}}\right\}^{2} d y^{2} \\
& +\left\{\frac{\sqrt{2} c-x^{2}+y^{2}}{x^{2}+y^{2}+2 z^{2}}\right\}^{2} d z^{2},
\end{aligned}
$$

where $c \in \mathbb{R}$. The principal curvatures of these systems, as well as Proposition 4.8, then show that all coordinate surfaces are Dupin cyclides.

Moreover, any Dupin cyclide is an isothermic surface, that is, it admits conformal curvature line coordinates. Hence, the associated systems of the 6-sphere coordinates consist of isothermic surfaces and are therefore isothermic triply orthogonal systems in the sense of Darboux [10].

Taking into account the explicit classification of isothermic Guichard nets given in $[28,29$, Chap. III], it follows that the associated systems of the 6-sphere coordinates do not admit a reparametrization into a Guichard net.

By (36), the Lamé coefficients of the dual systems of the 6-sphere coordinates read

$$
\begin{aligned}
& H_{1 c}^{\star}=\frac{\left(x^{2}+y^{2}+2 z^{2}\right)^{2}-\left(c+\sqrt{2}\left(y^{2}+z^{2}\right)\right)^{2}}{x^{2}+y^{2}+2 z^{2}}, \\
& H_{2 c}^{\star}=\frac{\left(x^{2}+y^{2}+2 z^{2}\right)^{2}-\left(c-\sqrt{2}\left(x^{2}+z^{2}\right)\right)^{2}}{x^{2}+y^{2}+2 z^{2}}, \\
& H_{3 c}^{\star}=\frac{-\sqrt{2}\left(x^{2}+y^{2}+2 z^{2}\right)^{2}-\sqrt{2}\left(c+\frac{1}{\sqrt{2}}\left(y^{2}-x^{2}\right)\right)^{2}}{x^{2}+y^{2}+2 z^{2}}
\end{aligned}
$$

and we obtain a 1-parameter family of new non-cyclic Guichard nets (Fig. 3). Since the seed Guichard net $f$ is totally cyclic, the torsion

$$
\tau_{i}^{\star}=\frac{\kappa_{j i}^{\star} \partial_{i} \kappa_{k i}^{\star}-\kappa_{k i}^{\star} \partial_{i} \kappa_{j i}^{\star}}{\left(\kappa_{j i}^{\star}\right)^{2}+\left(\kappa_{k i}^{\star}\right)^{2}}
$$

of any $x_{i}$-coordinate curve in the dual systems vanishes and therefore the dual Guichard nets consist of coordinate surfaces with planar curvature lines.

However, the dual systems do not contain parallel or totally umbilic surfaces, which is also reflected in the fact that not all associated systems of the dual systems are cyclic (cf.Proposition 4.8). Thus, the geometry of the associated systems of the original Guichard net and the associated systems of its dual systems change considerably.

\section{Bäcklund-type transformations of Guichard nets}

Classically [2, Sect. 4], two triply orthogonal systems form a Ribaucour pair if any two corresponding coordinate surfaces are Ribaucour transformations of each 

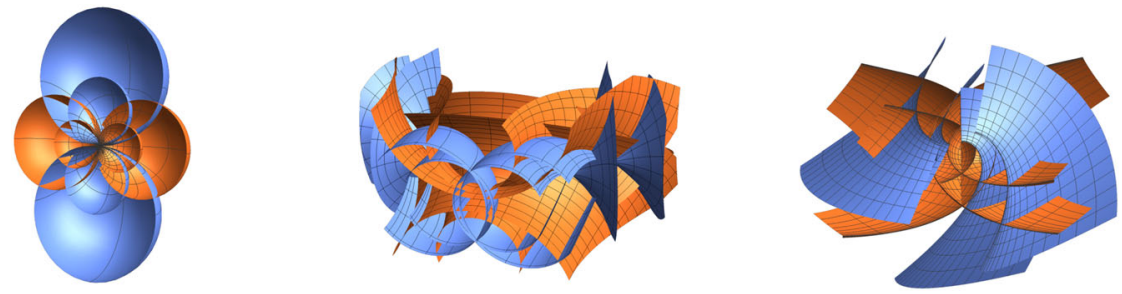

Fig. 3. The 6-sphere coordinates parametrized as Guichard net (left) with an associated system (middle) and the corresponding dual system (right)

other; that is, two corresponding surfaces envelop a common sphere congruence so that curvature lines correspond.

In this paper we are interested in Ribaucour transformations that preserve the Guichard condition of a Guichard net (cf. [2,3,23]).

To begin with, we summarize some facts about the Ribaucour transformation of triply orthogonal systems in general, which will then be used for the particular case of Guichard nets and their associated systems.

More details and results in this realm can be found in the two classical monographs $[2,3]$.

\subsection{Ribaucour transforms of triply orthogonal systems}

Any Ribaucour transform $\left(f^{\prime}, H_{1}^{\prime}, H_{2}^{\prime}, H_{3}^{\prime}\right)$ of a triply orthogonal system $\left(f, H_{1}\right.$, $\left.H_{2}, H_{3}\right)$ is uniquely described by four functions $\gamma_{1}, \gamma_{2}, \gamma_{3}$ and $\varphi$ satisfying

$$
\partial_{i} \gamma_{j}=\beta_{j i} \gamma_{i} \text { and } \partial_{i} \varphi=H_{i} \gamma_{i}
$$

where the data of the Ribaucour transform reads

$$
\begin{aligned}
f^{\prime} & =f-\frac{2 \varphi}{A}\left(\gamma_{1} N_{1}+\gamma_{2} N_{2}+\gamma_{3} N_{3}\right), \\
H_{i}^{\prime} & =H_{i}-\frac{2 \varphi \theta_{i}}{A}, \\
\beta_{i j}^{\prime} & =\beta_{i j}-\frac{2 \gamma_{i} \theta_{j}}{A}
\end{aligned}
$$

and

$$
\begin{aligned}
A & :=\gamma_{1}^{2}+\gamma_{2}^{2}+\gamma_{3}^{2}, \\
\theta_{i} & :=\partial_{i} \gamma_{i}+\beta_{j i} \gamma_{j}+\beta_{k i} \gamma_{k} .
\end{aligned}
$$

Here, $N_{i}$ denotes the unit normals of the coordinate surfaces $x_{i}=$ const of $f$.

Note that, by Lamé's equations, for three given functions $\gamma_{1}, \gamma_{2}$ and $\gamma_{3}$ satisfying (37) there exists a 1-parameter family of suitable functions $\varphi$, which define a 
1-parameter family of Ribaucour transforms. Since the radii of the enveloped Ribaucour sphere congruences for the coordinate surface family $x_{i}=$ const are given by

$$
R_{i}=-\frac{\varphi}{\gamma_{i}}
$$

the 1-parameter family of functions $\varphi$ indeed determines different Ribaucour transformations.

As observed by Bianchi in [2, Sect. 8], any Ribaucour transformation of a triply orthogonal system can be decomposed into two Combescure transformations and an inversion in the unit sphere. Since this construction will turn out to be a crucial tool in the transformation of Guichard nets, we recall it here in detail.

Suppose $f^{\prime}$ is a Ribaucour transformation of a triply orthogonal system $f$ described by the functions $\gamma_{1}, \gamma_{2}, \gamma_{3}$ and $\varphi$. If we denote the unit normals of the coordinate surfaces $x_{i}=$ const of $f$ by $N_{i}$, then the function

$$
\bar{f}:=\gamma_{1} N_{1}+\gamma_{2} N_{2}+\gamma_{3} N_{3}
$$

defines a Combescure transform $\bar{f}=: \mathcal{C}_{1}(f)$ of $f$ with induced metric

$$
\bar{I}=\theta_{1}^{2} d x^{2}+\theta_{2}^{2} d y^{2}+\theta_{3}^{2} d z^{2} .
$$

Moreover, the function $\bar{\varphi}:=\frac{1}{2}(A-1)$ fulfills condition (37) with respect to the induced metric $\bar{I}$ and therefore the functions $\left(\gamma_{1}, \gamma_{2}, \gamma_{3}, \bar{\varphi}\right)$ determine a Ribaucour transformation $\bar{f}^{\prime}$ of $\bar{f}$ :

$$
\bar{f}^{\prime}=\bar{f}-\frac{2 \bar{\varphi}}{A} \bar{f}=\frac{1}{A} \bar{f}=\frac{1}{|\bar{f}|^{2}} \bar{f} .
$$

Thus, this Ribaucour transformation is an inversion $\iota$ in the unit sphere and the induced metric of $\bar{f}^{\prime}=\left(\iota \circ \mathcal{C}_{1}\right)(f)$ is given by

$$
\bar{I}^{\prime}=\frac{1}{A^{2}} \bar{I} .
$$

Since the metric coefficients of $\bar{I}^{\prime}$ fulfill

$$
\partial_{j} \frac{\theta_{i}}{A}=\left\{\beta_{j i}-\frac{2 \gamma_{j} \theta_{i}}{A}\right\} \frac{\theta_{j}}{A}=\mathcal{R}\left(\beta_{j i}\right) \frac{\theta_{j}}{A},
$$

the transformed system $\left(\iota \circ \mathcal{C}_{1}\right)(f)$ is a Combescure transformation of $f^{\prime}$ and we indeed gain the sought-after decomposition of the Ribaucour transformation into two Combescure transformations and an inversion.

The constructed systems $\left(\bar{f}, \bar{f}^{\prime}\right)$ will be called the induced Combescure transformations of the Ribauocur pair $\left(f, f^{\prime}\right)$.

Conversely, a Combescure transformed system $\bar{f}$ of $f$ induces a 1-parameter family of Ribaucour transforms of $f$ : suppose that $\bar{f}$ is an arbitrary Combescure transform of $f$ and let $N_{i}$ denote the common unit normals of its coordinate surfaces $x_{i}=$ const. Then the functions

$$
\gamma_{i}:=\bar{f} \cdot N_{i}
$$


and a solution $\varphi$ of the integrable system

$$
\partial_{i} \varphi=H_{i} \gamma_{i}
$$

where $H_{i}$ are the metric coefficients of $f$, define a Ribaucour transform $f^{\prime}$ of $f$.

Since the function $\varphi$ is, by the Eq. (39), uniquely defined up to an additive constant, we obtain a 1-parameter family of induced Ribaucour transforms.

Furthermore, since the Ribaucour transformed rotational coefficients $\beta_{i j}^{\prime}$ only depend on the functions $\gamma_{1}, \gamma_{2}$ and $\gamma_{3}$, two Ribaucour transforms that are induced by the same system $\bar{f}$ share the same rotational coefficients. Hence, we obtain the following lemma (cf. Definition 2.2):

Lemma 5.1. If two Ribaucour transforms $f_{1}^{\prime}$ and $f_{2}^{\prime}$ are induced by the same triply orthogonal system, then $f_{1}^{\prime}$ and $f_{2}^{\prime}$ are related by a Combescure transformation.

\subsection{Bäcklund-type transformations of Guichard nets}

The purpose of this subsection is to geometrically understand the interplay between Ribaucour pairs of Guichard nets and the induced Combescure transforms in the decomposition discussed in the previous paragraphs.

Proposition 5.2. Let $\left(f, f^{\prime}\right)$ be a Ribaucour pair of Guichard nets, then the induced Combescure transforms $\bar{f}$ and $\bar{f}^{\prime}$ are $\alpha^{2}|\bar{f}|^{2}$ - and $\alpha^{2}\left|\bar{f}^{\prime}\right|^{2}$-systems, $\alpha \in \mathbb{R}$, respectively.

Proof. By [3, Sect. 11], we know that a Ribaucour pair $\left(f, f^{\prime}\right)$ of Guichard nets is generated by the data $\left(\gamma_{1}, \gamma_{2}, \gamma_{3}, \varphi\right)$ fulfilling

$$
\begin{aligned}
\partial_{j} \gamma_{i} & =\beta_{i j} \gamma_{j}, \quad \theta_{i}=\alpha \bar{\gamma}_{i} \\
\partial_{j} \bar{\gamma}_{i} & =\beta_{j i} \bar{\gamma}_{j}, \quad \bar{\theta}_{i}=\alpha \gamma_{i}, \\
A:=\gamma_{1}^{2}+\gamma_{2}^{2}+\gamma_{3}^{2} & =\bar{\gamma}_{1}^{2}+\bar{\gamma}_{2}^{2}-\bar{\gamma}_{3}^{2}=: \bar{A},
\end{aligned}
$$

where $\bar{\theta}_{i}:=\varepsilon_{i} \partial_{i} \bar{\gamma}_{i}+\varepsilon_{j} \beta_{i j} \bar{\gamma}_{j}+\varepsilon_{k} \beta_{i k} \bar{\gamma}_{k}$ and

$$
\varphi:=\frac{1}{\alpha}\left(\varepsilon_{i} H_{i} \bar{\gamma}_{i}+\varepsilon_{j} H_{j} \bar{\gamma}_{j}+\varepsilon_{k} H_{k} \bar{\gamma}_{k}\right)
$$

Therefore, the Lamé coefficients $\left(\theta_{1}, \theta_{2}, \theta_{3}\right)$ of the induced Combescure transform

$$
\bar{f}=\gamma_{1} N_{1}+\gamma_{2} N_{2}+\gamma_{3} N_{3}
$$

satisfy the condition

$$
\theta_{1}^{2}+\theta_{2}^{2}-\theta_{3}^{2}=\alpha^{2} \bar{A}=\alpha^{2} A=\alpha^{2}|\bar{f}|^{2} .
$$

Moreover, taking into account the change of the induced metric after an inversion in the unit sphere, completes the proof. 
Conversely, we can utilize this fact to construct Ribaucour pairs of Guichard nets and obtain in this way a Bäcklund-type transformation for Guichard nets:

Theorem 5.3. Let $\left(f, H_{1}, H_{2}, H_{3}\right)$ be a Guichard net and $\left(\bar{f}, \bar{H}_{1}, \bar{H}_{2}, \bar{H}_{3}\right)$ a Combescure transformed $\alpha^{2}|\bar{f}|^{2}$-system of $f$. Then the family of Ribaucour transformations of $f$ induced by $\bar{f}$ contains exactly one Guichard net $\mathcal{R}(f)$, which is given by the data

$$
\begin{gathered}
\mathcal{R}(f)=f-\frac{2 \varphi}{|\bar{f}|^{2}} \bar{f}, \\
\mathcal{R}\left(H_{i}\right)=H_{i}-\frac{2 \varphi}{|\bar{f}|^{2}} \bar{H}_{i},
\end{gathered}
$$

where $\varphi:=\frac{1}{\alpha^{2}}\left\{H_{1} \bar{H}_{1}+H_{2} \bar{H}_{2}-H_{3} \bar{H}_{3}\right\}$.

This Ribaucour transformation is said to be a Bäcklund-type transformation of $f$ with respect to the system $\bar{f}$.

Proof. Let $\bar{f}$ be a Combescure related $\alpha^{2}|\bar{f}|^{2}$-system of the Guichard net $f$. Then, by using the differentiated Guichard condition, we conclude that the 1-parameter family of induced Ribaucour transformations of $f$ are defined by the functions $\gamma_{i}:=\bar{f} \cdot N_{i}$ and

$$
\varphi_{\lambda}:=\frac{1}{\alpha^{2}}\left\{H_{1} \bar{H}_{1}+H_{2} \bar{H}_{2}-H_{3} \bar{H}_{3}\right\}+\lambda, \quad \lambda \in \mathbb{R},
$$

which satisfy the conditions (37). Therefore, these Ribaucour transforms are described by

$$
\begin{aligned}
\mathcal{R}(f) & =f-\frac{2 \varphi_{\lambda}}{A}\left(\gamma_{1} N_{1}+\gamma_{2} N_{2}+\gamma_{3} N_{3}\right)=f-\frac{2 \varphi_{\lambda}}{|\bar{f}|^{2}} \bar{f} \\
\mathcal{R}\left(H_{i}\right) & =H_{i}-\frac{2 \varphi_{\lambda} \theta_{i}}{A}=H_{i}-\frac{2 \varphi_{\lambda}}{|\bar{f}|^{2}} \bar{H}_{i} .
\end{aligned}
$$

Moreover, the Lamé coefficients satisfy

$$
\begin{aligned}
& \mathcal{R}\left(H_{1}\right)^{2}+\mathcal{R}\left(H_{2}\right)^{2}-\mathcal{R}\left(H_{3}\right)^{2} \\
& \quad=\frac{4 \varphi^{2}}{|\bar{f}|^{2}}\left(\bar{H}_{1}^{2}+\bar{H}_{2}^{2}-\bar{H}_{3}^{2}\right)-\frac{4 \varphi}{|\bar{f}|^{2}}\left(H_{1} \bar{H}_{1}+H_{2} \bar{H}_{2}-H_{3} \bar{H}_{3}\right)=\frac{4 \alpha^{2} \lambda}{|\bar{f}|^{2}} \varphi .
\end{aligned}
$$

Thus, an induced Ribaucour transform determined by (44) is again a Guichard net if and only if $\lambda=0$.

By Proposition 5.2 and Theorem 5.3, we have reduced the Bäcklund-type transformation for Guichard nets to the existence of Combescure transformed $\alpha^{2}|\bar{f}|^{2}$ systems of the Guichard nets. Thus, after an $\alpha^{2}|\bar{f}|^{2}$-system is determined, the Bäcklund-type transformation of any Combescure related Guichard net is obtained by simple computations from (43). 


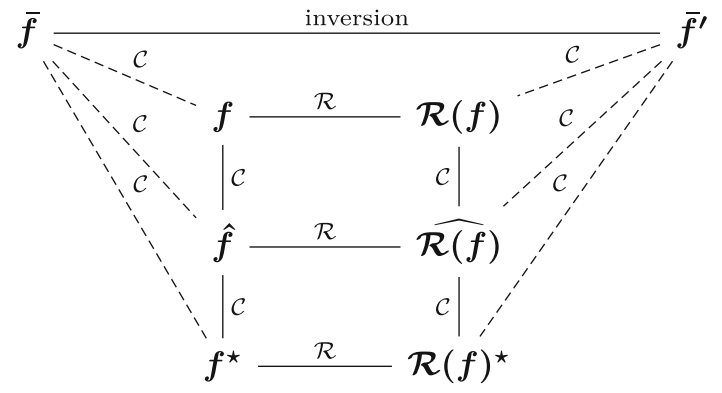

Fig. 4. Permutability theorem for a Guichard net $f$ with associated system $\hat{f}$ and dual system $f^{\star}$, where all Ribaucour transformations $\mathcal{R}$ are induced by the $\alpha^{2}|\bar{f}|^{2}$-system $\bar{f}$ and $\alpha^{2}\left|\bar{f}^{\prime}\right|^{2}$-system $\bar{f}^{\prime}$, respectively

Analogous proofs as given for Proposition 5.2 and Theorem 5.3 lead to a Bäcklundtype transformation for the class of $\lambda$-systems, where $\lambda \in \mathbb{R}$ is a constant:

Corollary 5.4. Let $\left(f, H_{1}, H_{2}, H_{3}\right)$ be a $\lambda$-system, $\lambda \in \mathbb{R}$, then any Ribaucour transformed $\lambda$-system $\mathcal{R}(f)$ of $f$ is induced by a Combescure related $\alpha^{2}|\bar{f}|^{2}$ system $\left(\bar{f}, \bar{H}_{1}, \bar{H}_{2}, \bar{H}_{3}\right)$ and determined by

$$
\begin{gathered}
\mathcal{R}(f)=f-\frac{2 \varphi}{|\bar{f}|^{2}} \bar{f}, \\
\mathcal{R}\left(H_{i}\right)=H_{i}-\frac{2 \varphi}{|\bar{f}|^{2}} \bar{H}_{i},
\end{gathered}
$$

where $\varphi:=\frac{1}{\alpha^{2}}\left\{H_{1} \bar{H}_{1}+H_{2} \bar{H}_{2}-H_{3} \bar{H}_{3}\right\}$.

Based on these observations, we prove a permutability theorem for the associated and dual systems of a Guichard net and the Bäcklund-type transformation (Fig. 4):

Theorem 5.5. Let $f$ be a Guichard net with associated systems $\hat{f}_{c}$ and dual systems $f_{c}^{\star}$. Then the Bäcklund-type transforms $\mathcal{R}\left(\hat{f}_{c}\right)$ and $\mathcal{R}\left(f_{c}^{\star}\right)$ induced by an $\alpha^{2}|\bar{f}|^{2}-$ system provide associated and dual systems of the Ribaucour transformed Guichard net $\mathcal{R}(f)$ induced by the same $\alpha^{2}|\bar{f}|^{2}$-system:

$$
\left.\mathcal{R}\left(\hat{f}_{c}\right)=\widehat{\mathcal{R}(f)}\right)_{c} \text { and } \mathcal{R}\left(f_{c}^{\star}\right)=\mathcal{R}(f)_{c}^{\star} .
$$

Proof. Firstly observe that, due to Lemma 5.1, the systems $\mathcal{R}(f), \mathcal{R}\left(\hat{f}_{c}\right)$ and $\mathcal{R}\left(f_{c}^{\star}\right)$ are again Combescure transformations of each other, because they are induced by the same $\alpha^{2}|\bar{f}|^{2}$-system.

Furthermore, let us denote the Lamé coefficients of the Guichard net $f$ and an associated system $\hat{f}$ with the corresponding dual system $f^{\star}$ by $\left(H_{i}\right)_{i},\left(\hat{H}_{i}\right)_{i}$ and $\left(H_{i}^{\star}\right)_{i}$, respectively. Thus, by Theorem 4.1 , the Lamé coefficients satisfy

$$
H_{i} H_{j}^{\star}+H_{j} H_{i}^{\star}=-2 \hat{H}_{i} \hat{H}_{j} .
$$


and we obtain the following relations

$$
\begin{aligned}
\varphi H_{i}^{\star}+\varphi^{\star} H_{i}+2 \hat{\varphi} \hat{H}_{i} & =\frac{2}{\alpha^{2}} \bar{H}_{j}, \\
\varphi \varphi^{\star}+\hat{\varphi}^{2} & =\frac{1}{\alpha^{2}}|\bar{f}|^{2},
\end{aligned}
$$

where

$$
\varphi=\frac{1}{\alpha^{2}} \sum \varepsilon_{i} H_{i} \bar{H}_{i}, \quad \hat{\varphi}=\frac{1}{\alpha^{2}} \sum \varepsilon_{i} \hat{H}_{i} \bar{H}_{i} \text { and } \varphi^{\star}=\frac{1}{\alpha^{2}} \sum \varepsilon_{i} H_{i}^{\star} \bar{H}_{i}
$$

These functions, together with the functions $\gamma_{i}=f \cdot N_{i}, \hat{\gamma}_{i}=\hat{f} \cdot N_{i}$ and $\gamma_{i}^{\star}=$ $f^{\star} \cdot N_{i}$, determine the induced Ribaucour transforms $\mathcal{R}(f), R\left(\hat{f}_{c}\right)$ and $\mathcal{R}\left(f_{c}^{\star}\right)$ (see Theorem 5.3 and Corollary 5.4).

A straightforward computation using the relations (47) then shows that the Lamé coefficients satisfy the conditions

$$
\mathcal{R}\left(H_{i}\right) \mathcal{R}\left(H_{j}^{\star}\right)-\mathcal{R}\left(H_{j}\right) \mathcal{R}\left(H_{i}^{\star}\right)=-2 \mathcal{R}\left(\hat{H}_{i}\right) \mathcal{R}\left(\hat{H}_{j}\right) .
$$

Thus, by Theorem 4.1, the systems $R\left(\hat{f}_{c}\right)$ and $\mathcal{R}\left(f_{c}^{\star}\right)$ form an associated and a dual system of $R(f)$ and the claim is therefore proven.

Acknowledgements Open access funding provided by Austrian Science Fund (FWF). This work originated in the author's doctoral thesis [29] and was partially supported by the FWF/JSPS Joint Project grant I1671- N26 Transformations and Singularities. Special gratitude is expressed to Udo Hertrich-Jeromin for helpful comments and various geometric insights into Guichard nets. Moreover, the author would like to thank Fran Burstall, Mason Pember and Wolfgang Schief for fruitful and enjoyable discussions on G- and O-surfaces.

Open Access This article is licensed under a Creative Commons Attribution 4.0 International License, which permits use, sharing, adaptation, distribution and reproduction in any medium or format, as long as you give appropriate credit to the original author(s) and the source, provide a link to the Creative Commons licence, and indicate if changes were made. The images or other third party material in this article are included in the article's Creative Commons licence, unless indicated otherwise in a credit line to the material. If material is not included in the article's Creative Commons licence and your intended use is not permitted by statutory regulation or exceeds the permitted use, you will need to obtain permission directly from the copyright holder. To view a copy of this licence, visit http://creativecommons.org/ licenses/by/4.0/.

\section{References}

[1] Bianchi, L.: Lezioni di Geometria Differenziale. Spoerri, Pisa (1894)

[2] Bianchi, L.: Le trasformazioni di Ribaucour dei sistemi $n$-pli ortogonali e il teorema generale di permutabilità. Ann. Mat. 3(27), 183-257 (1918)

[3] Bianchi, L.: Le trasformazioni di Ribaucour dei sistemi $n$-pli ortogonali e il teorema di permutabilità. Annali di Mat. 3(28), 187-234 (1919) 
[4] Burstall, F.E., Hertrich-Jeromin, U., Suyama, Y.: Curvilinear coordinates on generic conformally flat hypersurfaces and constant curvature 2-metrics. J. Math. Soc. Jpn. 70(2), 617-649 (2018)

[5] Calapso, P.: Alcune superficie di Guichard e le relative trasformazioni. Ann. Mat. 3(11), 201-251 (1904)

[6] Calapso, P.: Sulla teoria generale delle trasformazioni di Ribaucour e sue applicazioni alla generalizzazione delle trasformazioni di Darboux. Ann. Mat. 3(29), 17-70 (1920)

[7] Canevari, S., Tojeiro, R.: Hypersurfaces of two space forms and conformally flat hypersurfaces. Ann. Mat. Pura Appl. (4) 197(1), 1-20 (2018)

[8] Christoffel, E.: Ueber einige allgemeine Eigenschaften der Minimumsflächen. J. für die Reine Angew. Mat. 67, 218-228 (1867)

[9] Combescure, E.: Sur les déterminants fonctionnels et les coordonnées curvilignes. Ann. Sci. École Norm. Sup. 4, 93-131 (1867)

[10] Darboux, G.: Mémoire sur la théorie des coordonnées curvilignes, et des systèmes orthogonaux. Ann. de l'Éc. Norm. (2) 7, 275-348 (1878)

[11] Darboux, G.: Leçons sur les systèmes orthogonaux et les coordonnées curvilignes. Deuxième édition, Complétée. Gauthier-Villars, Paris (1910)

[12] Demoulin, A.: Sur les surfaces $\Omega$. R. Acad. Sci. Paris 153, 927-929 (1911)

[13] do Rei Filho, C., Tojeiro, R.: Minimal conformally flat hypersurfaces. J. Geom. Anal. 29(3), 2931-2956 (2019)

[14] dos Santos, J., Tenenblat, K.: The symmetry group of Lamé's system and the associated Guichard nets for conformally flat hypersurfaces. SIGMA Symmetry Integr. Geom. Methods Appl. 9, 033 (2013)

[15] Eisenhart, L.P.: Transformations of Surfaces. Princeton University Press, Princeton (1923)

[16] Eisenhart, L.P.: A Treatise on the Differential Geometry of Curves and Surfaces. Ginn, Boston (1960)

[17] Ganzha, E.I., Tsarev, S.P.: An algebraic formula for superposition and the completeness of the Bäcklund transformations of (2+1)-dimensional integrable systems. Russ. Math. Sur. 51(6), 1200-1202 (1996)

[18] Guichard, C.: Sur les surfaces isothermiques. Ann. Sci. École Norm. Sup. 130, 159-162 (1900)

[19] Guichard, C.: Sur les systèmes triplement indéterminés et sur les systèmes tripleorthogonaux, vol. 25. Gauthier-Villars. Scientia, Paris (1905)

[20] Hertrich-Jeromin, U.: On conformally flat hypersurfaces and Guichard's nets. Beitr. Algebra Geom. 35(2), 315-331 (1994)

[21] Hertrich-Jeromin, U., Suyama, Y.: Conformally flat hypersurfaces with cyclic Guichard net. Int. J. Math. 18(3), 301-329 (2007)

[22] Hertrich-Jeromin, U., Suyama, Y.: Conformally flat hypersurfaces with Bianchi-type Guichard net. Osaka J. Math. 50(1), 1-30 (2013)

[23] Hertrich-Jeromin, U., Suyama, Y., Umehara, M., Yamada, K.: A duality for conformally flat hypersurfaces. Beitr. Algebra Geom. 56(2), 655-676 (2015)

[24] Hertrich-Jeromin, U., Tjaden, E., Zürcher, M.: On Guichard's Nets and Cyclic Systems. Preprint FIM ETH Zürich (1997)

[25] Pember, M.: Lie applicable surfaces. Commun. Anal. Geom. (to appear)

[26] Salkowski, E.: Dreifach orthogonale Flächensysteme. Encykl. Math. Wiss. 3, 541-606 (1921)

[27] Schief, W., Konopelchenko, B.: On the unification of classical and novel integrable surfaces. I. Differential geometry. R. Soc. Lond. Proc. Math. Phys. Eng. Sci. 459(2029), 67-84 (2003) 
[28] Szewieczek, G.: Isothermic Guichard nets (in preparation)

[29] Szewieczek, G.: Conformally flat hypersurfaces and Guichard nets. Ph.D. thesis (2017)

[30] Tojeiro, R., Canevari, S.: The Ribaucour transformation for hypersurfaces of two space forms and conformally flat hypersurfaces. Bull. Braz. Math. Soc. (N.S.) 49(3), 593-613 (2018)

[31] Weatherburn, C.E.: Differential Geometry of Three Dimensions, vol. 1. Cambridge University Press, Cambridge (1927)

Publisher's Note Springer Nature remains neutral with regard to jurisdictional claims in published maps and institutional affiliations. 